\title{
Cholesterol metabolism in cardiac sarcoidosis
}

\section{Simonen, Piia}

2016-05

Simonen , P , Lehtonen , J , Gylling , H \& Kupari , M 2016 , ' Cholesterol metabolism in

cardiac sarcoidosis ' , Atherosclerosis , vol. 248 , pp. 210-215 . https://doi.org/10.1016/j.atherosclerosis.2016.03.018

http://hdl.handle.net/10138/223957

https://doi.org/10.1016/j.atherosclerosis.2016.03.018

publishedVersion

Downloaded from Helda, University of Helsinki institutional repository.

This is an electronic reprint of the original article.

This reprint may differ from the original in pagination and typographic detail.

Please cite the original version. 


\title{
Cholesterol metabolism in cardiac sarcoidosis ${ }^{\text {is }}$
}

\author{
Piia Simonen ${ }^{a}{ }^{*}$, Jukka Lehtonen ${ }^{a}$, Helena Gylling ${ }^{\text {b }}$, Markku Kupari a \\ ${ }^{a}$ University of Helsinki and Helsinki University Central Hospital, Heart and Lung Center, Division of Cardiology, P.O. BOX 340, FI-00029 HUS, Helsinki, \\ Finland \\ ${ }^{\mathrm{b}}$ University of Helsinki and Helsinki University Central Hospital, Division of Internal Medicine, P.O. BOX 700, FI-00029 HUS, Helsinki, Finland
}

\section{A R T I C L E I N F O}

\section{Article history:}

Received 12 January 2016

Received in revised form

7 March 2016

Accepted 16 March 2016

Available online 18 March 2016

\section{Keywords:}

Atherosclerosis

Campesterol

Cholesterol absorption

Cholesterol synthesis

Inflammation

Lathosterol

Myocardial disease

\begin{abstract}
A B S T R A C T
Background and aims: Patients with cardiac sarcoidosis (CS) suffer from myocardial inflammation, but atherosclerosis is not infrequent in these patients. However, the classical atherosclerotic risk factors, such as perturbed serum lipids and whole-body cholesterol metabolism, remain unravelled in CS.

Methods: We assessed serum non-cholesterol sterols, biomarkers of whole-body cholesterol synthesis and cholesterol absorption efficiency, with gas-liquid chromatography in 39 patients with histologically verified CS and in an age-adjusted random population sample $(\mathrm{n}=124)$.

Results: CS was inactive or responding to treatment in all patients. Concentrations of serum, LDL, and HDL cholesterol and serum triglycerides were similar in CS patients and in control subjects. Cholesterol absorption markers were higher in CS patients than in controls (eg serum campesterol to cholesterol ratio in CS $246 \pm 18$ vs in controls $190 \pm 810^{2} \times \mu \mathrm{mol} / \mathrm{mmol}$ of cholesterol, $\mathrm{p}=0.001$ ). Cholesterol synthesis markers were lower in CS patients than in controls (eg serum lathosterol to cholesterol ratio in CS $102 \pm 8$ vs in controls $195 \pm 510^{2} \mathrm{x} \mu \mathrm{mol} / \mathrm{mmol}$ of cholesterol, $\left.\mathrm{p}=0.000\right)$. In CS patients, cholesterol absorption markers significantly correlated with plasma prohormone brain natriuretic peptide (proBNP), a marker of hemodynamic load.

Conclusion: High cholesterol absorption efficiency, which is suggested to be atherogenic, characterized the metabolic profile of cholesterol in CS patients. The association between cholesterol absorption efficiency and plasma proBNP concentration, which suggests a link between inflammation, cholesterol homeostasis, and hemodynamic load, warrants further studies in order to confirm this finding and to reveal the underlying mechanisms.
\end{abstract}

() 2016 Elsevier Ireland Ltd. All rights reserved.

\section{Introduction}

Sarcoidosis is a multisystem inflammatory disease characterized by the formation of non-caseating granulomas in several tissues. Cardiac sarcoidosis (CS) may accompany the systemic sarcoidosis or, alternatively, may exist as an isolated form of disease. The baseline pathogenesis of the inflammation is unknown, but it is speculated that the chronic antigenic stimulation of unknown origin enhances the expression of $\mathrm{T}$ helper cell cytokines and chemokines [1]. Serum and lipoprotein lipid concentrations and their metabolism is in general affected by the activation of the acute

\footnotetext{
Part of this study has been presented as an abstract at the American Heart Association Scientific Sessions 2014, Chicago, Illinois.

* Corresponding author.

E-mail addresses: piia.simonen@hus.fi (P. Simonen), jukka.lehtonen@hus.fi (J. Lehtonen), helena.gylling@hus.fi (H. Gylling), markku.kupari@hus.fi (M. Kupari).
}

phase response caused by inflammation. Changes in lipoproteins towards atherogenicity and an increased risk of atherosclerosis are well documented consequences of inflammation eg in rheumatoid arthritis [2]. In addition to myocardial inflammatory involvement, simultaneous coronary artery disease (CAD) can also occur in sarcoidosis [3-5]. The lesions in the coronary arteries can result from the sarcoidosis-caused vasculitis, but the possible role of atherosclerosis cannot be ruled out [5]. Very little is known about serum and lipoprotein lipids or their atherogenicity in sarcoidosis. In the few studies which have been carried out in patients with active systemic sarcoidosis, LDL cholesterol concentration was not elevated compared with control subjects [6-8]. HDL cholesterol concentration was reduced in patients with active sarcoidosis, but it was normalized during the corticosteroid therapy [9]. However, nothing is known about the whole-body cholesterol metabolism, whose profile may contribute to the development of atherosclerosis and CAD [10]. Accordingly, we were interested to see, whether serum and lipoprotein lipids and cholesterol metabolism are 
disturbed in patients with CS to find out, if the risk of atherosclerosis is increased in these patients. We assessed the hypothesis that cholesterol absorption and synthesis are abnormal in CS. Thus, we evaluated cholesterol metabolism by determining serum noncholesterol sterols, valid biomarkers of cholesterol synthesis and cholesterol absorption efficiency [11-20] in patients with CS and in an age-adjusted random population sample from the Finnish Population Register Centre.

\section{Materials and methods}

\subsection{Study population}

The study population consisted of 39 patients with histologically verified CS and of 124 controls of similar age randomly selected from the Finnish Population Register Centre and living in Helsinki. All CS diagnoses were made in our hospital after year 2008 using the criteria described in our previous works [21,22]. In brief, a histology of non-caseating granulomatous inflammation in endomyocardial biopsy or in extracardiac tissue sample associated with clinical manifestations and findings at cardiac imaging compatible with CS was mandatory for the diagnosis. The clinical data of these patients including demographics, medical history and symptoms, laboratory tests, electrocardiogram, imaging results, and histopathological analyses of biopsy material were retrospectively reviewed and analysed in detail for the present study. These patients were followed up regularly as ambulatory outpatients in our hospital. The use of lipid lowering medication was an exclusion criteria for both CS patients and control subjects. Any immunosuppressive medication had to remain unchanged for one month before the study.

All subjects gave their written informed consent. The study was performed according to the principles of the Declaration of Helsinki. The Ethics Committee for the Department of Medicine, Hospital District of Helsinki and Uusimaa approved the study protocol.

\subsection{Study design}

All subjects were studied on their normal habitual diet. Fasting blood samples were drawn after a 12- hour fast. The subjects were weighed and their height was measured, and a history of previous diseases and current drug treatment was recorded. The CS patients were also medically evaluated as part of their regular ambulatory clinical follow-up.

\subsection{Laboratory methods and measurements}

Laboratory measurements of the CS patients included assessments of blood count, serum creatinine, serum alanine aminotransferase, thyroid stimulating hormone, serum thyroxine, plasma high-sensitive C-reactive protein (hsCRP), plasma troponin $\mathrm{T}$, plasma prohormone brain natriuretic peptide (proBNP), and plasma glucose, and they were analysed with routine standardized methods at the Central Laboratory of Helsinki University Central Hospital (HUSLAB). Serum total, LDL, and HDL cholesterol and serum triglycerides were determined both from the CS patients and control subjects, and they were analysed enzymatically by using an automated analyzer system (HUSLAB).

Serum cholesterol, cholesterol precursors (squalene, cholestenol ( $5 \alpha$-cholest-8-en- $3 \beta$-ol), desmosterol, and lathosterol), plant sterols (sitosterol and campesterol), and cholestanol, a metabolite of cholesterol, were quantified with capillary gas-liquid chromatography (GLC) with a 50-m Ultra 2 capillary column, as described previously [23]. Serum concentrations of non-cholesterol sterols were expressed in $\mu \mathrm{g} / \mathrm{dl}$ and also as the ratio to cholesterol $\left(10^{2} \mathrm{x}\right.$ $\mu \mathrm{mol} / \mathrm{mmol}$ of cholesterol) by adjusting the non-cholesterol sterol concentrations to the cholesterol value of the same GLC run, and multiplying the ratio by $10^{2}$ in order to get rid of the decimals. Because the assessment of absolute whole-body cholesterol metabolism is difficult and laborious, it is generally accepted that the ratios of serum non-cholesterol sterols to cholesterol are valid biomarkers of cholesterol metabolism [24,25]. Accordingly, the ratios of serum cholesterol precursors to cholesterol reflect cholesterol synthesis [11-16], while the ratios of plant sterols and cholestanol reflect cholesterol absorption efficiency [14,16-20]. We also calculated the lathosterol/campesterol ratio, which reflects the whole-body cholesterol metabolism [14], and campesterol/cholestanol ratio, which is a biomarker of dietary plant sterol intake [26].

\subsection{Statistics}

Statistical analyses were performed with SPSS for Windows 19.0 statistics program (SPSS, Chicago, IL, USA). The normality and the homogeneity of variance assumptions were checked before further analyses. The comparisons for continuous variables between CS patients and control subjects were performed with Student's t-test. The variables not normally distributed even after logarithmic transformation, non-homogenous in variance, or non-continuous were tested with Mann-Whitney $U$ test and Fisher exact test. Spearman correlation coefficients were calculated. A $p$-value of $<0.05$ was considered statistically significant. The results are given as mean \pm SEM.

\section{Results}

\subsection{Demographics and clinical characteristics}

The main clinical characteristics of the study population are demonstrated in Table 1 . The CS patients comprised 31 women and 8 men, and the mean age of the patients was 53.1 years (range, 37-69 years). The control group consisted of 124 subjects (61 women, 63 men), who were randomly selected from population cohorts. The CS patients did not differ from the controls regarding age or body mass index (BMI), but the female preponderance in the CS patients was statistically significant $(\mathrm{p}=0.0009)$. However, there was no difference in demographics and clinical characteristics between men and women in the CS group.

The inflammatory and cardiac/hemodynamic markers and plasma glucose were not analysed in the control subjects, because they were clinically in good health, and only one subject had type 2 diabetes with oral hypoglycemic treatment.

CS with clinically isolated cardiac involvement, defined as cardiac involvement with neither previous history or any current signs or symptoms of evident extracardiac sarcoidosis according to clinical examination, chest $\mathrm{x}$-ray and laboratory tests, was observed in $28 / 39$ patients (72\%), whereas the remaining 11 patients had already known extracardiac sarcoidosis (mainly pulmonary sarcoidosis; skin or extrathoracic lymph nodes were affected in solitary cases, and liver was affected in one patient). The demographic and clinical characteristics and medication were similar in patients with isolated CS and CS with extracardiac manifestations.

The histology of sarcoidosis had been confirmed by endomyocardial biopsy $(\mathrm{n}=20)$ or by biopsy of mediastinal lymph nodes $(\mathrm{n}=11)$, lungs $(\mathrm{n}=5)$, skin $(\mathrm{n}=2)$ or liver $(\mathrm{n}=1)$.

The mean duration of CS from diagnosis was 28 months (range 5-52 months) at the time of our study. The mean ejection fraction was $50.7 \pm 1.8 \%$ in the CS patients (reference value $>50.0 \%$ ). Blood count, kidney function and liver enzymes were normal in all CS patients. Troponin $\mathrm{T}$ was elevated in six patients, whereas hsCRP 
Table 1

Demographics and clinical characteristics of the study population.

\begin{tabular}{lll}
\hline Variables & Control subjects, $\mathrm{n}=124$ & Cardiac sarcoidosis, $\mathrm{n}=39$ \\
\hline Gender, $\mathrm{F} / \mathrm{M}, \mathrm{n}$ & $61 / 63$ & $31 / 8$ \\
Age, yrs & $52.3 \pm 0.2$ & $53.1 \pm 1.4$ \\
$\mathrm{BMI}, \mathrm{kg} / \mathrm{m}^{2}$ & $26.8 \pm 0.5$ & $25.7 \pm 0.9$ \\
Plasma hsCRP, mg/l & $\mathrm{ND}$ & $1.43 \pm 0.18$ \\
Plasma troponin T, ng/l & $\mathrm{ND}$ & $9.82 \pm 0.97$ \\
Plasma proBNP, ng/l & $\mathrm{ND}$ & $618 \pm 125$ \\
Plasma glucose, mmol/l & $\mathrm{ND}$ & $5.38 \pm 0.09$ \\
Immunosuppressive treatment, $\mathbf{n}$ & & 9 \\
$\quad$ None & 124 & 8 \\
$\quad$ Corticosteroids & 0 & 14 \\
$\quad$ AZA/MTX & 0 & 8 \\
$\quad$ Corticosteroid + AZA/MTX & 0 & 36 \\
Betablocking agent, $\mathbf{n}$ & 10 &
\end{tabular}

Mean \pm SEM. hsCRP $=$ high sensitive C-reactive protein; proBNP $=$ prohormone brain natriuretic peptide; AZA $=$ azathioprine; MTX = methotrexate; ND = not determined.

Reference values for hsCRP $<3 \mathrm{mg} / \mathrm{l}$; troponin $\mathrm{T}<15 \mathrm{ng} / \mathrm{l}$; proBNP females $<222 \mathrm{ng} / \mathrm{l}$, males $<194 \mathrm{ng} / \mathrm{l}$.

Difference between cardiac sarcoidosis patients and control subjects: gender: $\mathrm{p}=0.000$, age: NS, BMI: NS.

was normal. At the time of the study, the plasma concentrations of proBNP were normal in 18 patients, whereas they were elevated but stable or declining in 21 patients when compared with their previous outpatient data.

Nine CS patients had no immunosuppressive treatment, whereas 8 patients were on corticosteroids, 13 had azathioprine, one patient had methotrexate, and 8 patients had the combination treatment of corticosteroids and azathioprine or methotrexate (Table 1). Thirty-six patients used beta-blocking agents. Gender, age, BMI, routine laboratory test results, or length or activity of the disease did not differ between the different treatment groups. Ten control subjects used beta-blocking agents.

\subsection{Serum and lipoprotein lipids and serum non-cholesterol sterols}

Serum and lipoprotein lipid concentrations were similar between the CS patients and control subjects (Table 2). In both groups, serum and lipoprotein lipid and serum non-cholesterol sterol values were independent of gender. Furthermore, serum and lipoprotein lipids and serum non-cholesterol sterols were similar between the different immunosuppressive treatment groups in the CS patients. Beta-adrenergic blockers had no effect on serum and lipoprotein lipid and serum non-cholesterol sterol values in the CS patients or control subjects.

Regarding the serum cholesterol precursors, the ratios of squalene and lathosterol to cholesterol were significantly lower in the CS patients compared with the control subjects (Table 2). This difference could be observed in both women and men (eg lathosterol to cholesterol ratio $96 \pm 7$ in CS women vs $196 \pm 710^{2} \mu \mathrm{mol} /$ mmol of cholesterol in control women, $\mathrm{p}=0.001$, and $125 \pm 22$ in CS men vs $193 \pm 2410^{2} \mu \mathrm{mol} / \mathrm{mmol}$ of cholesterol in control men, $\mathrm{p}=0.031)$. On the other hand, the ratios of serum campesterol, sitosterol, and cholestanol were significantly higher in the CS patients compared with the control group. The difference was again present in both women and men (eg sitosterol to cholesterol ratio $149 \pm 10$ for CS women vs $123 \pm 610^{2} \times \mu \mathrm{mol} / \mathrm{mmol}$ of cholesterol in control women $(\mathrm{p}=0.022)$, and $140 \pm 9$ in CS men vs $116 \pm 7$ in control men, $(\mathrm{p}=0.016)$, respectively. The lathosterol/campesterol ratio was lower in the CS patients compared with the control subjects. The significant difference was present in both gender. Campesterol/cholestanol ratio was identical between the groups reflecting similar plant sterol intake.

In order to confirm that the cholesterol synthesis and absorption markers were operating as expected, the synthesis markers as well as the absorption markers were interrelated. Serum cholesterol precursors correlated with each other expressed as concentrations and ratios to cholesterol in the CS patients and in the control subjects as shown for cholestenol and lathosterol concentrations in Table 3. Likewise, serum plant sterols and cholestanol correlated with each other as concentrations and ratios to cholesterol as

Table 2

Serum and lipoprotein lipids and serum non-cholesterol sterols in the study population.

\begin{tabular}{|c|c|c|c|}
\hline Variables & $\begin{array}{l}\text { Control subjects, } \\
\mathrm{n}=124\end{array}$ & $\begin{array}{l}\text { Cardiac sarcoidosis, } \\
\mathrm{n}=39\end{array}$ & P Value \\
\hline Serum cholesterol, $\mathrm{mmol} / \mathrm{L}$ & $6.12 \pm 0.55$ & $5.65 \pm 0.16$ & 0.195 \\
\hline LDL cholesterol, mmol/L & $4.01 \pm 0.36$ & $3.34 \pm 0.13$ & 0.098 \\
\hline HDL cholesterol, mmol/L & $1.47 \pm 0.13$ & $1.48 \pm 0.11$ & 0.891 \\
\hline Serum triglycerides, $\mathrm{mmol} / \mathrm{L}$ & $1.43 \pm 0.13$ & $1.75 \pm 0.17$ & 0.179 \\
\hline \multicolumn{4}{|c|}{ Cholesterol precursors, $\mathbf{1 0}^{2} \times \mu \mathrm{mol} / \mathrm{mmol}$ of cholesterol } \\
\hline Squalene & $32.3 \pm 1.0$ & $14.1 \pm 1.2$ & 0.000 \\
\hline Cholestenol & $14.0 \pm 0.7$ & $16.3 \pm 0.9$ & 0.086 \\
\hline Desmosterol & $75.9 \pm 1.4$ & $81.7 \pm 3.7$ & 0.092 \\
\hline Lathosterol & $194.8 \pm 4.8$ & $101.7 \pm 7.5$ & 0.000 \\
\hline \multicolumn{4}{|c|}{ Plant sterols and cholestanol, $\mathbf{1 0}^{2} \times \mu \mathrm{mol} / \mathrm{mmol}$ of cholesterol } \\
\hline Campesterol & $190.0 \pm 7.5$ & $245.5 \pm 17.7$ & 0.001 \\
\hline Sitosterol & $121.6 \pm 3.8$ & $144.2 \pm 10.1$ & 0.011 \\
\hline Cholestanol & $139.2 \pm 3.0$ & $154.6 \pm 5.2$ & 0.012 \\
\hline Campesterol/cholestanol, $\mu \mathrm{g} / \mu \mathrm{g}$ & $1.39 \pm 0.05$ & $1.56 \pm 0.08$ & 0.101 \\
\hline Lathosterol/campesterol, $\mu \mathrm{g} / \mu \mathrm{g}$ & $1.28 \pm 0.07$ & $0.52 \pm 0.06$ & 0.000 \\
\hline
\end{tabular}

Mean \pm SEM. 
Table 3

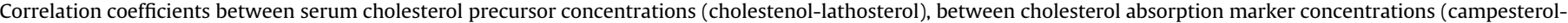
cholestanol), and between cholesterol precursor and absorption marker concentrations and ratios to cholesterol (lathosterol-cholestanol) in the study population.

\begin{tabular}{|c|c|c|c|c|}
\hline Variables & $\begin{array}{l}\text { Control subjects, } \\
\mathrm{n}=124\end{array}$ & P Value & $\begin{array}{l}\text { Cardiac sarcoidosis, } \\
\mathrm{n}=39\end{array}$ & P Value \\
\hline Cholestenol-lathosterol, $\mu \mathrm{g} / \mu \mathrm{g}$ & +0.788 & 0.000 & +0.902 & 0.000 \\
\hline Campesterol-cholestanol, $\mu \mathrm{g} / \mu \mathrm{g}$ & +0.398 & 0.000 & +0.810 & 0.000 \\
\hline Lathosterol-cholestanol, $\mu \mathrm{g} / \mu \mathrm{g}$ & +0.037 & 0.686 & -0.128 & 0.436 \\
\hline $\begin{array}{l}\text { Lathosterol-cholestanol, } \\
10^{2} \times \mu \mathrm{mol} / \mathrm{mmol} \text { of cholesterol }\end{array}$ & -0.538 & 0.000 & -0.458 & 0.003 \\
\hline
\end{tabular}

shown for campesterol and cholestanol concentrations. To assess whether cholesterol homeostasis was intact cholesterol precursors were related to cholesterol absorption markers (Table 3). Cholesterol precursor sterols correlated with plant sterols and cholestanol in the CS patients and in the control subjects but only as ratios to cholesterol as shown for lathosterol-cholestanol. The correlation between synthesis and absorption markers was not significant if the sterols were expressed as concentrations.

In the CS patients, serum lipids and non-cholesterol sterols were not associated with plasma hsCRP or troponin $\mathrm{T}$ concentrations. However, plasma proBNP correlated positively with the cholestanol $(\mathrm{r}=0.323, \mathrm{p}=0.032)$ and campesterol to cholesterol ratios $(\mathrm{r}=0.351, \mathrm{p}=0.025)$.

\section{Discussion}

The novel finding in this study was that cholesterol metabolism was different in CS patients compared with the control subjects in spite of similar serum and lipoprotein lipid concentrations. Irrespective of gender, serum cholesterol precursors were low and plant sterols and cholestanol were elevated in the CS patients compared with the control subjects. Importantly, in both groups all cholesterol precursor sterols as well as plant sterols and cholestanol were interrelated and cholesterol homeostasis was intact which suggested that the serum biomarkers adequately reflected endogenous cholesterol metabolism. Therefore, it seems obvious that cholesterol synthesis is low and cholesterol absorption efficiency is upregulated in CS confirming our hypothesis. The second novel finding was that cholesterol absorption efficiency was related to plasma proBNP, which is a marker of hemodynamic load.

The CS study population was biopsy-verified, and, according to the clinical evaluation, cardiac echocardiography, and laboratory tests, the disease was inactive or responding to treatment. Because there was no difference in serum lipid and non-cholesterol sterol levels in patients with or without immunosuppressive therapy or by the different immunosuppressants, it seems evident that the medication was not a confounding factor related to cholesterol metabolism. The CS patients and the control subjects were well matched regarding to age and BMI, and the female preponderance in the CS group had no effect on the results.

In the present study, which included CS patients with the disease being inactive or responding to treatment, serum and lipoprotein lipids did not differ between the CS patients and the control subjects. These results are comparable to the findings in an earlier study including inactive/treated sarcoidosis patients without cardiac involvement [7]. In patients with untreated or active sarcoidosis HDL cholesterol concentration was low [7,9], but during corticosteroid therapy it returned to normal levels [9]. Low HDL cholesterol concentration in active sarcoidosis may result from elevated serum amyloid A (SAA) concentration [27], which can displace apo A-I from HDL exposing the lipoprotein particle to rapid clearance. Recently, studies of HDL proteome have raised the possibility of a link between SAA, cholesterol efflux capacity, inflammation and atherosclerosis [28].

Serum cholesterol precursors, plant sterols, and cholestanol are valid biomarkers of whole-body cholesterol metabolism [11-20,24,25]. To be certain that they operated also in CS as expected, we interrelated the cholesterol precursors with each other in both groups as well as the plant sterols and cholestanol. Cholesterol homeostasis was checked by relating the precursor sterols to the plant sterols and cholestanol. Interestingly, the synthesis marker sterols were significantly interrelated either as concentrations or ratios to cholesterol, and the same was observed for the absorption markers. However, the synthesis markers correlated with the absorption markers only when assessed as ratios to cholesterol. This is a new observation, and the reason for this discrepancy may be that the non-cholesterol sterols are carried in different lipoproteins. The distribution of non-cholesterol sterol concentrations in different lipoproteins resembles that of cholesterol [29]. However, when adjusted to cholesterol concentration of the lipoprotein, the synthesis markers are mainly carried in LDL, whereas the absorption markers are mainly carried in $\operatorname{HDL}[29,30]$. Accordingly, since the synthesis markers are transported in the same lipoproteins, they were interrelated in spite of being expressed as concentrations or as ratios to cholesterol, and the same was true for the absorption markers. It is noteworthy that when assessing cholesterol homeostasis, the markers are carried in different lipoproteins and have to be expressed as ratios to cholesterol.

In the present study, high cholesterol absorption efficiency and low endogenous synthesis of cholesterol characterized the profile of cholesterol metabolism in the CS patients. It seems obvious that it is the disease process itself which regulates the metabolic profile. To the best of our knowledge, there is no earlier information regarding cholesterol metabolism in CS or in systemic sarcoidosis, or, on the whole, in other chronic inflammatory diseases. Thus, we have no reference to cholesterol metabolism in inflammatory diseases in general. Cholesterol absorption efficiency is increased in patients with type 1 diabetes [31] as in the CS patients in the present study, but the detailed mechanism is unknown. Since plasma proBNP and cholesterol absorption were slightly but significantly interrelated, we assume that either the chronic inflammation and/or the hemodynamic load in CS is able to increase cholesterol absorption efficiency, but the possible mechanism remains open. Regarding the regulation of the two main intestinal sterol transporters, Niemann-Pick C1-Like 1 (NPC1L1) is mainly activated by the lack of cellular cholesterol, whereas excess cellular cholesterol and oxysterols activate the ABCG5 and ABCG8 efflux transporters. In addition to the cellular cholesterol content, genetic variants of both transporters interfere with cholesterol absorption efficiency [20,32-34] so that high cholesterol absorption increased the risk of CAD [32,33], whereas low cholesterol absorption protected from CAD [20,34]. Even though there is evidence that high plasma proBNP concentration is associated with elevated cardiovascular risk [35], the possible link between circulating proBNP concentration and regulation of cholesterol 
absorption efficiency remains open and warrants further evaluation.

In conclusion, whole-body cholesterol metabolism was perturbed in the CS patients so that cholesterol absorption efficiency was elevated and cholesterol synthesis was low. High cholesterol absorption efficiency is considered to be atherogenic, so that the profile of cholesterol metabolism in CS contributes to atherogenic potential. The association between sterol absorption and plasma proBNP concentration, which suggests a link between inflammation, cholesterol homeostasis and hemodynamic load, warrants further evaluation.

\section{Funding}

No external funding.

\section{Conflicts of interest}

The authors declare no conflict of interest.

\section{Statement of originality}

The manuscript is original and has not been published previously, except in the form of an abstract (American Heart Association Scientific Sessions 2014, Chicago, Illinois), and is not under consideration for publication elsewhere.

\section{Author contributions}

PS, JL, HG and MK participated in the conception and design of the study. PS collected the data, performed the statistical analyses and interpreted the data. PS and HG were responsible for the noncholesterol sterol analyses. PS wrote the manuscript. All authors reviewed and edited the manuscript, and approved the final version to be submitted.

\section{Acknowledgements}

Ms Leena Kaipiainen is acknowledged for excellent technical assistance.

\section{References}

[1] E.S. Chen, D.R. Moller, Etiologies of sarcoidosis, Clin. Rev. Allergy Immunol. 49 (2015) 6-18.

[2] M. Amezaga Urruela, M.E. Suarez-Almazor, Lipid paradox in rheumatoid arthritis: changes with rheumatoid arthritis therapies, Curr. Rheumatol. Rep. 14 (2012) 428-437.

[3] V. Singh, S. Luthra, R. Kouides, A.K. Gadir, What's wrong with this artery? A medical disease discovered by a surgeon, BMJ Case Rep. (2014), http:// dx.doi.org/10.1136/bcr-2014-205645.

[4] J.C. Hansen, A.R. Patel, H.N. Nayak, J.D. Moss, N. Sweiss, J.F. Beshai, Cardiac sarcoidosis and coronary artery disease: a two-hit mechanism to left ventricular dysfunction (Or is it?), Sarcoidosis Vasc. Diffuse Lung Dis. 30 (2013) $237-240$.

[5] T. Egashira, S. Makino, A. Kunitomi, M. Okada, Y. Maekawa, K. Fukuda, Necessity for rule out coronary artery disease with the positive findings of ${ }^{18} \mathrm{~F}-$ FDG-PET in case of systemic sarcoidosis, Int. J. Cardiol. 172 (2014) e401-e402.

[6] L.S. Kindman, H.S. Gilbert, J.S. Almenoff, H. Ginsberg, R. Fagerstrom, A.S. Teirstein, High-density lipoprotein cholesterol is reduced in patients with sarcoidosis, Am. J. Med. 86 (1989) 376-378.

[7] A. Salazar, J. Maña, X. Pinto, J.M. Argimon, M.J. Castiñeiras, C. Fiol, R. Pujol, Low levels of high density lipoprotein cholesterol in patients with active sarcoidosis, Atherosclerosis 136 (1998) 133-137.

[8] J. Ivanišević, J. Kotur-Stevuljević, A. Stefanović, Z. Jelić-Ivanović, S. Spasić, V. Videnović-Ivanov, J. Ilić, Dyslipidemia and oxidative stress in sarcoidosis patients, Clin. Biochem. 45 (2012) 677-682.

[9] A. Salazar, J. Mañá, X. Pintó, J.M. Argimón, L. Hurtado, R. Pujol, Corticosteroid therapy increases HDL-cholesterol concentrations in patients with active sarcoidosis and hypoalphalipoproteinemia, Clin. Chim. Acta 320 (2002) $59-64$.
[10] T.A. Miettinen, H. Gylling, T. Strandberg, S. Sarna, Baseline serum cholestano as predictor of recurrent coronary events in subgroup of Scandinavian simvastatin survival study. Finnish 4S investigators, BMJ 316 (1998) 1127-1130.

[11] T.A. Miettinen, Serum squalene and methyl sterols as indicators of cholesterol synthesis in vivo, Life Sci. 8 (1969) 713-721.

[12] I. Björkhem, T. Miettinen, E. Reihnér, S. Ewerth, B. Angelin, K. Einarsson, Correlation between serum levels of some cholesterol precursors and activity of HMG-CoA reductase in human liver, J. Lipid Res. 28 (1987) 1137-1143.

[13] H.J. Kempen, J.F. Glatz, J.A. Gevers Leuven, H.A. van der Voort, M.B. Katan, Serum lathosterol concentration is an indicator of whole-body cholesterol synthesis in humans, J. Lipid Res. 29 (1988) 1149-1155.

[14] T.A. Miettinen, R.S. Tilvis, Y.A. Kesäniemi, Serum plant sterols and cholesterol precursors reflect cholesterol absorption and synthesis in volunteers of a randomly selected male population, Am. J. Epidemiol. 131 (1990) 20-31.

[15] W.C. Duane, Serum lathosterol levels in human subjects reflect changes in whole body cholesterol synthesis induced by lovastatin but not dietary cholesterol, J. Lipid Res. 36 (1995) 343-348.

[16] P. Simonen, H. Gylling, T.A. Miettinen, The validity of serum squalene and non-cholesterol sterols as surrogate markers of cholesterol synthesis and absorption in type 2 diabetes, Atherosclerosis 197 (2008) 883-888.

[17] T.A. Miettinen, R.S. Tilvis, Y.A. Kesäniemi, Serum cholestanol and plant stero levels in relation to cholesterol metabolism in middle-aged men, Metabolism 38 (1989) 136-140.

[18] T. Sudhop, D. Lütjohann, A. Kodal, M. Igel, D.L. Tribble, S. Shah, I. Perevozskaya K. von Bergmann, Inhibition of intestinal cholesterol absorption by ezetimibe in humans, Circulation 106 (2002) 1943-1948.

[19] X. Lin, S.B. Racette, M. Lefevre, L. Ma, C.A. Spearie, K. Steger-May, R.E. Ostlund Jr., Combined effects of ezetimibe and phytosterols on cholestero metabolism. A randomized, controlled feeding study in humans, Circulation 124 (2011) 596-601.

[20] J.C. Cohen, A. Pertsemlidis, S. Fahmi, S. Esmail, G.L. Vega, S.M. Grundy H.H. Hobbs, Multiple rare variants in NPC1L1 associated with reduced sterol absorption and plasma low-density lipoprotein levels, Proc. Natl. Acad. Sci. U. S. A. 103 (2006) 1810-1815.

[21] R. Kandolin, J. Lehtonen, M. Graner, J. Schildt, K. Salmenkivi, S.M. Kivistö M. Kupari, Diagnosing isolated cardiac sarcoidosis, J. Intern Med. 270 (2011) 461-468.

[22] R. Kandolin, J. Lehtonen, J. Airaksinen, T. Vihinen, H. Miettinen, K. Ylitalo, K. Kaikkonen, S. Tuohinen, P. Haataja, T. Kerola, J. Kokkonen, M. Pelkonen, P. Pietilä-Effati, S. Utriainen, M. Kupari, Cardiac sarcoidosis: epidemiology, characteristics and outcome over 25 years in a nationwide study, Circulation 131 (2015) 624-632.

[23] T.A. Miettinen, Cholesterol metabolism during ketoconazole treatment in man, J. Lipid Res. 29 (1988) 43-51.

[24] S.M. Grundy, Plasma noncholesterol sterols as indicators of cholesterol absorption, J. Lipid Res. 54 (2013) 873-875.

[25] H. Gylling, J. Plat, S. Turley, H.N. Ginsberg, L. Ellegård, W. Jessup, P.J. Jones, D. Lütjohann, W. Maerz, L. Masana, G. Silbernagel, B. Staels, J. Borén, A.L. Catapano, G. De Backer, J. Deanfield, O.S. Descamps, P.T. Kovanen, G. Riccardi, L. Tokgözoglu, M.J. Chapman, European Atherosclerosis Society Consensus Panel on phytosterols, plant sterols and plant stanols in the management of dyslipidaemia and prevention of cardiovascular disease, Atherosclerosis 232 (2014) 346-360.

[26] X. Lin, S.B. Racette, L. Ma, M. Wallendorf, C.A. Spearie, R.E. Ostlund Jr., Plasma biomarker of dietary phytosterol intake, PLoS One 10 (2) (2015) e0116912. http://dx.doi.org/10.1371/journal.pone.0116912.

[27] A. Salazar, J. Maña, C. Fiol, I. Hurtado, J.M. Argimon, R. Pujol, X. Pinto, Influence of serum amyloid A on the decrease of high density lipoprotein-cholesterol in active sarcoidosis, Atherosclerosis 152 (2000) 497-502.

[28] M.J. Thomas, M.G. Sorci-Thomas, SAA: a link between cholesterol efflux capacity and inflammation? J. Lipid Res. 56 (2015) 1519-1530.

[29] P.P. Simonen, H. Gylling, T.A. Miettinen, The distribution of squalene and noncholesterol sterols in lipoproteins in type 2 diabetes, Atherosclerosis 194 (2007) 222-229.

[30] R.S. Tilvis, T.A. Miettinen, Serum plant sterols and their relation to cholesterol absorption, Am. J. Clin. Nutr. 43 (1986) 92-97.

[31] T.A. Miettinen, H. Gylling, J. Tuominen, P. Simonen, V. Koivisto, Low synthesis and high absorption of cholesterol characterize type 1 diabetes, Diabetes Care 27 (2004) 53-58.

[32] D. Teupser, R. Baber, U. Ceglarek, M. Scholz, T. Illig, C. Gieger, L.M. Holdt A. Leichtle, K.H. Greiser, D. Huster, P. Linsel-Nitschke, A. Schäfer, P.S. Braund, L. Tiret, K. Stark, D. Raaz-Schrauder, G.M. Fiedler, W. Wilfert, F. Beutner, S. Gielen, A. Großhennig, I.R. König, P. Lichtner, I.M. Heid, A. Kluttig, N.E. El Mokhtari, D. Rubin, A.B. Ekici, A. Reis, C.D. Garlichs, A.S. Hall, G. Matthes, C. Wittekind, C. Hengstenberg, F. Cambien, S. Schreiber, K. Werdan, T. Meitinger, M. Loeffler, N.J. Samani, J. Erdmann, H.E. Wichmann, H. Schunkert, J. Thiery, Genetic regulation of serum phytosterol levels and risk of coronary artery disease, Circ. Cardiovasc Genet. 3 (2010) 331-339.

[33] G. Silbernagel, M.J. Chapman, B. Genser, M.E. Kleber, G. Fauler, H. Scharnagl, T.B. Grammer, B.O. Boehm, K.M. Mäkelä, M. Kähönen, R. Carmena, E.R. Rietzschel, E. Bruckert, J.E. Deanfield, T.A. Miettinen, O.T. Raitakari, T. Lehtimäki, W. März, High intestinal cholesterol absorption is associated with cardiovascular disease and risk alleles in ABCG8 and ABO: evidence from the LURIC and YFS cohorts and from a meta-analysis, J. Am. Coll. Cardiol. 62 (2013) 291-299. 
[34] The Myocardial Infarction Genetics Consortium Investigators, Inactivating mutations in NPC1L1 and protection from coronary heart disease, N. Engl. J. Med. 371 (2014) 2072-2082.

[35] E. Di Angelantonio, R. Chowdhury, N. Sarwar, K.K. Ray, R. Gobin, D. Saleheen,
A. Thompson, V. Gudnason, N. Sattar, J. Danesh, B-type natriuretic peptides and cardiovascular risk. Systemic review and meta-analysis of 40 prospective studies, Circulation 120 (2009) 2177-2187. 